\title{
Effects of noise near a homoclinic bifurcation in an electrochemical system
}

\author{
Gerardo J. Escalera Santos, ${ }^{1}$ M. Rivera, ${ }^{1}$ M. Eiswirth, ${ }^{2}$ and P. Parmananda ${ }^{1}$ \\ ${ }^{1}$ Facultad de Ciencias, UAEM, Avenida Universidad 1001, Colonia Chamilpa, Cuernavaca, Morelos, Mexico \\ ${ }^{2}$ Fritz-Haber-Institut der Max-Planck-Gesellschaft, Faradayweg 4-6, D-14195 Berlin, Germany
}

(Received 2 March 2004; published 26 August 2004)

\begin{abstract}
The interaction of external noise with an electrochemical oscillator (anodic dissolution of iron) has been studied experimentally on both sides of the homoclinic bifurcation. In the oscillatory regime the regularity of the limit cycle behavior was destroyed with increasing noise amplitude until no periodicity was observable any more. In contrast, the response of the excitable state (fixed point regime) became more regular at intermediate noise levels.
\end{abstract}

DOI: 10.1103/PhysRevE.70.021103

PACS number(s): 05.40.-a, 05.45.Xt

\section{INTRODUCTION}

From the earliest days, physicists and engineers, especially working in the field of electronics and communications, have considered noise a nuisance and have tried to minimize it. However, due to the pioneering works of Benzi et al. [1,2] and Nicolis and Nicolis [3], the scientific community was introduced to the concept of stochastic resonance (SR). This involves the inception of noise generated resonances that are generic in nature and therefore found in a number of diverse natural processes [4-7]. The SR trend has really caught on and consequently a large number of theoretical and experimental papers have appeared extending the notion of SR and discovering interesting applications of noise as in (1) detection of weak signals [8-11], (2) transmission of information in sensory neurons [12-14], (3) induction of regularity in excitable systems [15], and (4) maintaining traveling waves in sub excitable media [16]. All these examples seem to indicate that noise, if used judiciously, can play a constructive role in various processes.

The notion of excitability comes from biology [17] and chemistry $[18,19]$. The dynamics of a system are labeled as excitable if a small perturbation to a state space variable can induce a large excursion of the variable in state space. This behavior has its origin in the shape of the nullclines for the equations that dictate the system dynamics. It is relevant in a number of important fields; for example, in neurophysiology, the underlying excitability of neurons is deemed crucial in efforts to understand information processing by an individual neuron or neural networks [23-25].

Recently, noise induced order was reported for excitable systems [15]. This phenomenon, termed coherence resonance (CR) by the authors, explains how purely stochastic fluctuations (white noise with a Gaussian distribution) of a system parameter are capable of invoking regular dynamics. The normalized variance (NV) [15] and the coherence factor $(\beta)$ [26] are two common measures used to quantify the induced regularity. More recently, the emergence of CR was verified for a nonexcitable chemical system using simulations and experiments performed in the vicinity of a supercritical Hopf bifurcation [27].

Here we present experimental results involving the interaction of external noise with nonlinear chemical dynamics.
We include both the destructive and constructive roles of noise in chemical systems. Corresponding numerical simulations yield consistent results. The constructive role of noise is the emergence of CR when the system is exhibiting excitable fixed point behavior. This is in contrast with the results of Ref. [18] (apart from being a different chemical system), which report CR for nonexcitable dynamics.

\section{EXPERIMENTAL SETUP}

The experimental system is a three-electrode electrochemical cell set up to study the potentiostatic (EG\&G PARC model 362 potentiostat) electrodissolution of iron in a mixture of copper sulfate and sulfuric acid. The anode was an iron disk ( $5 \mathrm{~mm}$ diameter) shrouded by epoxy. The electrolyte solution was a mixture of $1.0 \mathrm{M}$ sulfuric acid and $0.4 M$ copper sulfate. A volume of about $500 \mathrm{ml}$ was maintained in the cell. The anodic potential $(V)$ measured relative to a saturated calomel reference electrode (SCE) was used as the control (bifurcation) parameter on to which the external noise was superimposed. The cathode was a $5 \mathrm{~mm}$ diameter copper rod shrouded by epoxy. Oscillations in the anodic current $I$ (the current between the anode and the cathode) were recorded using a 12-bit data acquisition card at a sampling frequency of $33 \mathrm{~Hz}$. The external noise used in the experiments was generated by another computer using a random number generator whose output, distributed in the interval $[-1,1]$, is consistent with white noise with a Gaussian distribution. This output is converted to an analog signal and superimposed on the anodic voltage via the potentiostat. The frequency at which the noise amplitude is varied at about $\approx 1.7 \mathrm{~Hz}$. A schematic of the experimental setup is shown in Fig. 1.

Before studying the noise provoked dynamics, the autonomous system behavior was explored. By varying the anodic voltage $(V)$ as the bifurcation parameter, different dynamical responses of the observable (anodic current $I$ ) were mapped out [19]. Other system parameters like the electrolyte concentration and temperature were maintained constant.

Figure 2 shows the experimental bifurcation diagram constructed using the anodic voltage $(V)$ as the bifurcation parameter. It needs to be pointed out that, although the absolute values of the different bifurcation (transition) points may 


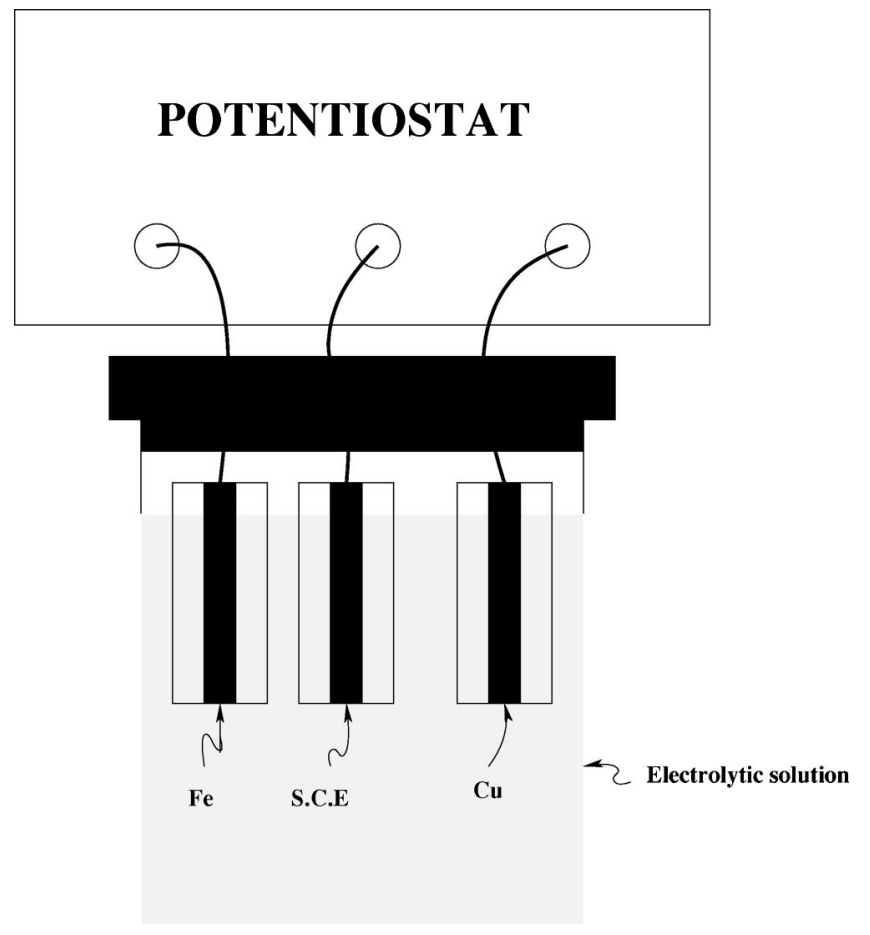

FIG. 1. A schematic representation of the electrochemical cell with iron, copper, and saturated calomel electrodes being the anode, cathode, and reference, respectively.

vary, the qualitative structure of the diagram is robust and therefore reproducible. The two main types of dynamics observed were fixed point behavior (constant current response) and period-1 oscillations emerging from a supercritical Hopf bifurcation at $\approx 175 \mathrm{mV}$. Right after the supercritical Hopf bifurcation harmonic, fast oscillations were found. For increasing voltage, typical relaxation oscillations resulted, the period of which became increasingly large. In order to quan-

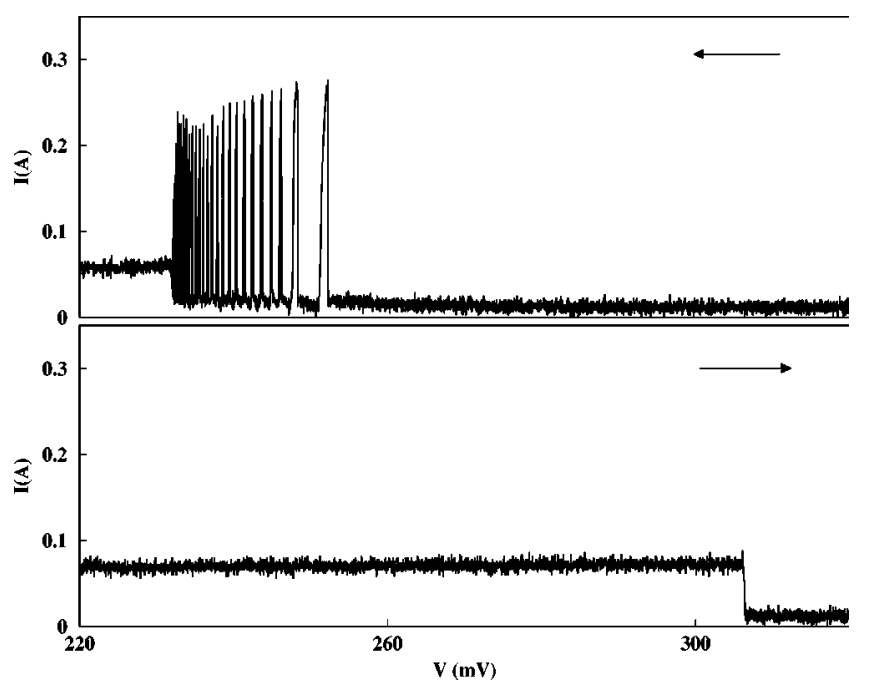

FIG. 2. Experimental bifurcation diagram constructed by varying (increasing and decreasing) the system parameter $(V)$. It shows that the parameter space is divided into two dynamical domains, namely, the fixed point behavior and autonomous periodic oscillations. tify the underlying bifurcation resulting in the emergence of the excitable fixed point behavior at the upper end of the bifurcation diagram of Fig. 2, we analyzed the increment in the period $(T)$ of the autonomous period-1 oscillations as a function of the anodic voltage as shown in Fig. 3(a). This dramatic augmentation of the time period of the autonomous oscillatory behavior is consistent with the existence of a homoclinic bifurcation in the vicinity. Moreover, Fig. 3(b) indicates that the experimental $\ln \left(V-V_{h}\right)$ vs $T$ curve can be fitted by a straight line, where $V_{h}$ is the bifurcation point separating relaxation oscillations and the excitable fixed point behavior. The results of Fig. 3 indicate that the oscillations seem to get slower until they die out in a homoclinic bifurcation at $V_{h}$.

To summarize, at potential values slightly below $175 \mathrm{mV}$, a stable focus type fixed point response was found, whereas for values of anodic voltages slightly above $240 \mathrm{mV}$, excitable fixed point dynamics exist. Moreover, for the purposes of the experiments reported here, the set point for the autonomous system was above and below the homoclinic bifurcation point $\left(V_{h}\right)$.

\section{DESTRUCTIVE ROLE OF NOISE}

The destructive role of noise has been studied rather exhaustively in the literature [20-22] from a theoretical standpoint. For the purposes of our experiments, the set point was chosen in the oscillatory domain of the autonomous dynamics near the homoclinic bifurcation. The system, for this set of parameter values, exhibits period-1 spiking behavior in the anodic current $(I)$. These dynamics were subjected to stochastic fluctuations of the anodic voltage $(V)$ given by $V$ $=V_{0}+A \xi$ where $V_{0}$ is the set-point anodic voltage, $\xi$ is the superimposed noise (white with a Gaussian distribution), and $A$ is the amplitude of the added noise. Figure 4 shows the variations in the system response as $A$ is increased monotonically. Three observed time series of anodic current $(I)$ and their corresponding power (Fourier) spectra are shown. Time series (a) shows the autonomous dynamics of the period-1 spiking behavior with an intrinsic frequency of $0.079 \mathrm{~Hz}$ [curve (a) of the Fourier spectra]. When the noise is switched on the peak corresponding to the characteristic frequency decreases and the spectrum widens [curve (b) of the Fourier spectra]. For increasing noise strength, the characteristic frequency peak disappears [curve (c) of the spectra] and the power spectrum becomes flat. These experimental results are consistent with previously reported numerical simulations [26] and can be explained as follows: For an intermediate noise level the spikes retain their characteristic shape (i.e., essentially follow the deterministic path), while there is a finite distribution of periods. This is attributed to the fact that only during a certain phase of the oscillation is the limit cycle sufficiently sensitive to the fluctuations. Higher noise levels can trigger spikes at practically all phases; hence all periodicity is destroyed, and a continuous train of spikes results. 

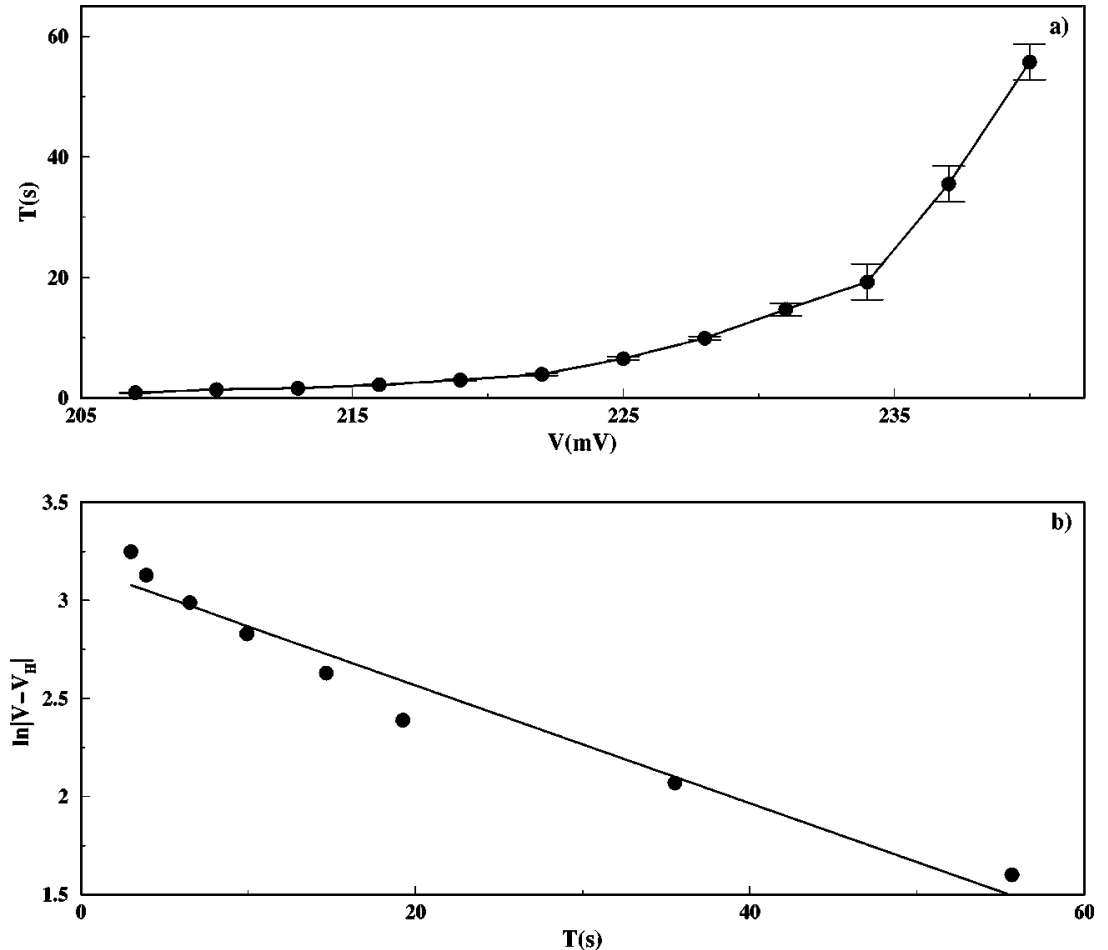

FIG. 3. Analyzing in detail the autonomous dynamics at the upper end of the bifurcation diagram. (a) Incrementing time period $(T)$ of the autonomous period-1 behavior as the anodic voltage $(V)$ is increased. (b) The $\ln \left(V-V_{h}\right)$ vs $T$ curve can be fitted by a straight line indicating an underlying homoclinic bifurcation.

\section{CONSTRUCTIVE ROLE OF NOISE: COHERENCE RESONANCE}

\section{A. Experimental results}

For this set of experiments, the set point of the anodic voltage $V_{0}$ is chosen such that the anodic current $I$ exhibits excitable fixed point behavior. In order to minimize the effect of parameter drifts, a point quite far from the homoclinic bifurcation was chosen. Consequently, large noise amplitudes were required. Subsequently, superimposed noise on the anodic voltage was increased and the provoked dynamics analyzed. The normalized variance was used to quantify the extent of induced regularity. It is defined as $\sqrt{\operatorname{var}\left(t_{p}\right) /\left\langle t_{p}\right\rangle}$, where $t_{p}$ is the time between successive peaks. It is evident that the more regular the dynamics the lower the value of the computed NV. For purely periodic dynamics the NV approaches 0 . Figure 5 shows the experimental NV curve as a function of noise amplitude $A$. Corresponding time series for three extreme points of the NV curve are also shown. The first relative maximum [label (a) of the NV curve] corresponds to low levels of noise where the activation threshold is seldom crossed, generating a sparsely populated irregular spike sequence. As the noise amplitude is further increased, the NV decreases, reaching a minimum (b) corresponding to
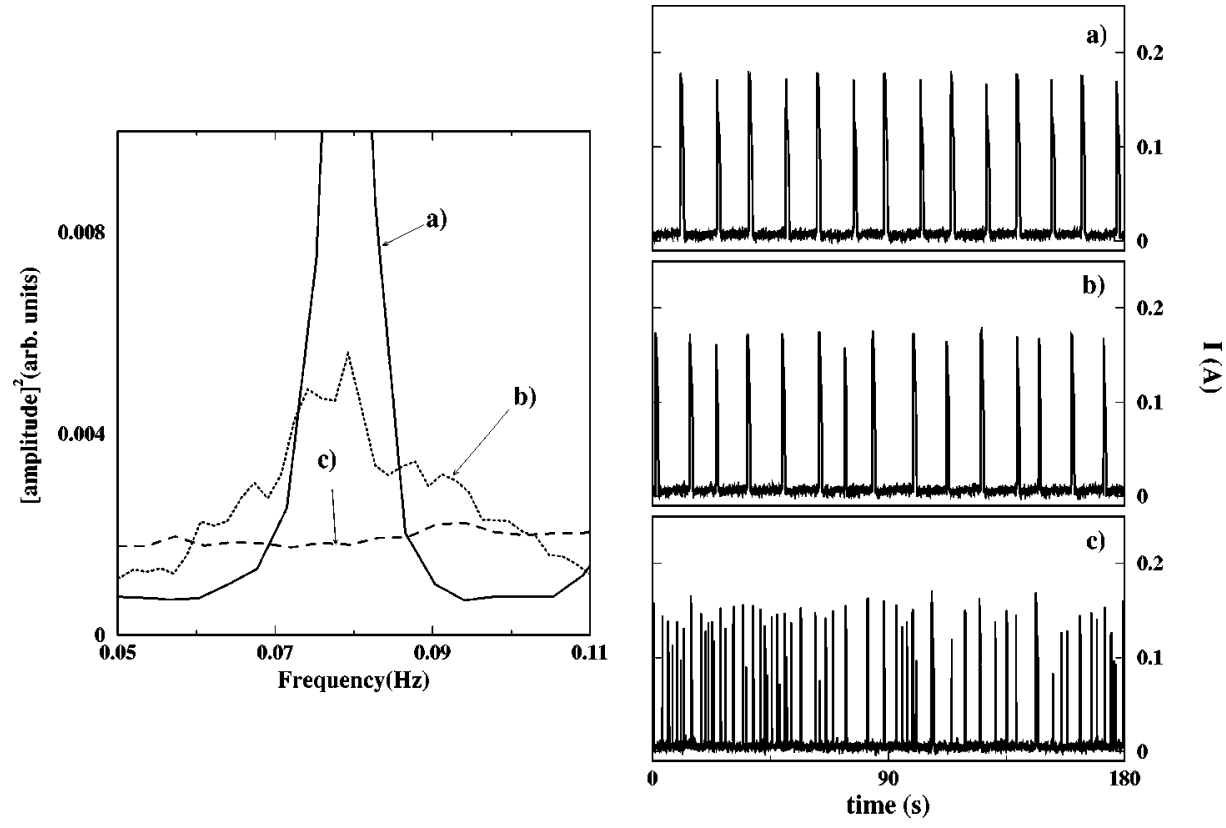

FIG. 4. Destruction of the limit cycle behavior: $V_{0}=187 \mathrm{mV}$. Time series of the anodic current $I$ for three different amplitudes of the superimposed noise (a) $A$ $=0 \mathrm{mV}$, (b) $A=20 \mathrm{mV}$ and (c) $A$ $=95 \mathrm{mV}$. The corresponding power spectra reveal the effect of noise. 

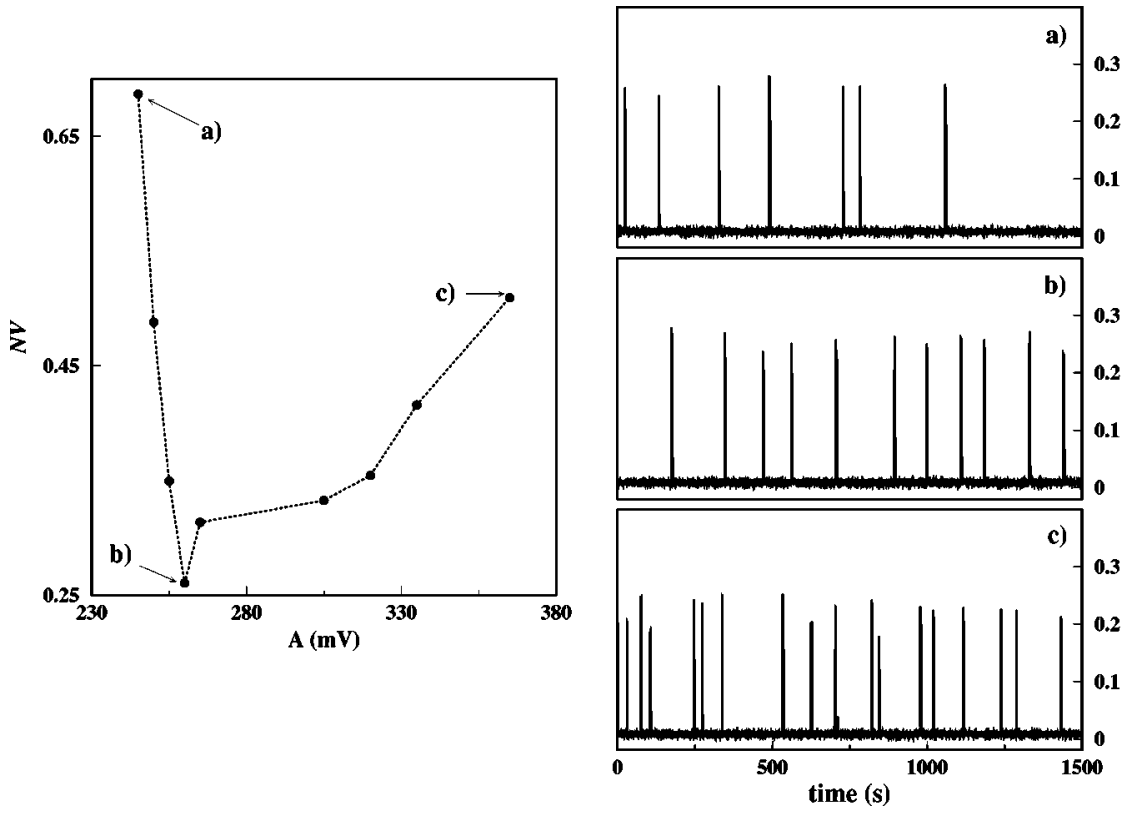

FIG. 5. Emergence of coherence resonance: $V_{0}=400 \mathrm{mV}$. The experimental normalized variance curve as the noise amplitude $(A)$ is monotonically increased. Corresponding time series of the anodic current (I) for points (a) $A$ $=245 \mathrm{mV}$, (b) $A=260 \mathrm{mV}$, (c) $A$ $=365 \mathrm{mV}$ of the $\mathrm{NV}$ curve are also shown.

an optimum noise level where maximum regularity of the generated spike sequence is observed. The emergence of this minimum, labeled CR in the literature [15], is a result of the interplay between stochastic fluctuations and the excitatory and refractory times of spikes. As the amplitude of superimposed noise is increased further, the observed regularity is destroyed as is manifested by an increase in the NV [label (c) of the NV curve]. This is a consequence of the dynamics being dominated by noise.

Figure 6 shows another set of experiments, performed at a slightly different set point of the anodic voltage. These also show the emergence of CR in the excitable chemical system. The observance of CR in excitable systems is attributed to a complicated interplay of superimposed noise with the two characteristic times of the provoked spikes, namely, the activation time and the excursion time. It has been verified quantitatively [15] that for small noise amplitudes the acti- vation time contribution dominates, whereas for large noise amplitudes it is the contribution of the excursion time that is dominant. Consequently, the optimum noise level, which corresponds to the minimum in the computed NV curve, is determined by the ratio of the two characteristic (activation and excursion) times.

\section{B. Numerical simulations}

To validate the experimental observations, we performed numerical simulations using the following two-dimensional numerical model [28] simulating the dynamics of an electrochemical cell under potentiostatic conditions:

$$
\epsilon \dot{u}=\frac{v-u}{R}-f(u, c),
$$
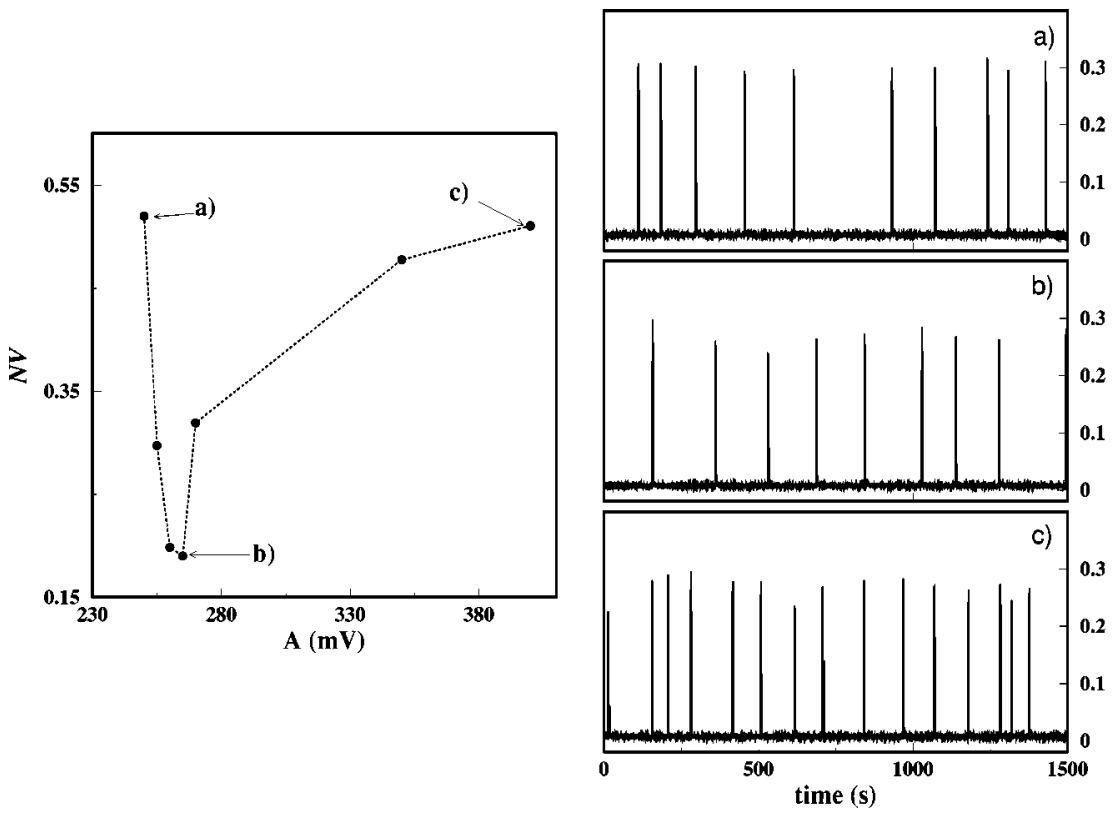

FIG. 6. Emergence of coherence resonance: $V_{0}=430 \mathrm{mV}$. The experimental normalized variance curve as the noise amplitude $(A)$ is monotonically increased. Corresponding timeseries of the anodic current (I) for points (a) $A$ $=250 \mathrm{mV}$, (b) $A=265 \mathrm{mV}$, (c) $A$ $=400 \mathrm{mV}$ of the NV curve are also shown. 


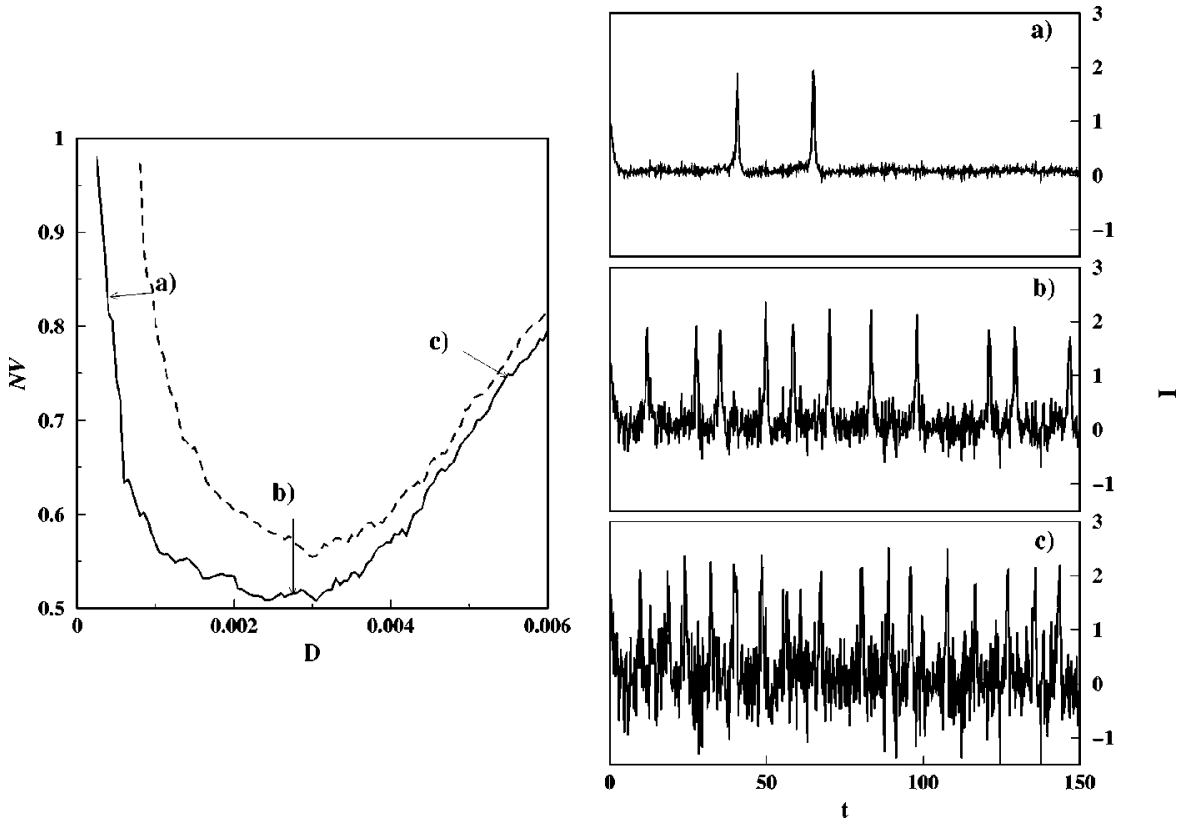

FIG. 7. Emergence of coherence resonance in the numerical model. Two normalized variance curves as the noise amplitude $(A)$ is monotonically increased for two different set points. The dashed curve corresponds to the set point $v_{0}=29.245$ while the full curve corresponds to the set point $v_{0}$ $=29.238$. Corresponding time series of the anodic current $(I)$ for points (a), (b), and (c) of the NV curve for $v_{0}=29.238$ are also shown. Consistent with experiments, the figure shows the inception of maximum regularity for an optimum level of noise.

$$
\dot{c}=\frac{u-v}{R}+(1-c)+\alpha f(u, c),
$$

where

$$
f(u, c)=c\left(a_{1} u+a_{2} u^{2}+a_{3} u^{3}\right) .
$$

In the above set of equations the two variables $u(t)$ and $c(t)$ correspond to the electrode potential and the surface concentration, respectively. The three system parameters $\epsilon$, $R$, and $v$ represent the double layer capacitance, the Ohmic resistance, and the applied potential. Equation (1) represents the conservation of charge, whereas Eq. (2) corresponds to the mass balance equation. The anodic current, defined as $(v-u) / R$, is our observable for the model dynamics. Details of the model system, including underlying bifurcations, are discussed in detail and characterized elsewhere [28]. Dynamical behavior similar to that in the experiments is observed for appropriate model parameters, i.e., for $\epsilon=0.03$, $\alpha=0.1, R=10, a_{1}=1.125, a_{2}=-0.075$, and $a_{3}=0.00125$. The anodic voltage $v$, the bifurcation parameter, is subsequently varied to map out different model responses [28]. For values $28.097 \leqslant v \leqslant 29.235$ a limit cycle behavior exists, which disappears such that a stable fixed point behavior is observed for $v>29.235$. Similar to the experiments, noise was superimposed on the anodic voltage $(v)$ in the parameter domain where the autonomous dynamics exhibit fixed point behavior $(v>29.235)$.

The applied potential $v$ was perturbed stochastically, $v$ $=v_{0}+D \xi$, where $\xi$ represents Gaussian white noise with zero mean and unit standard deviation. This set of stochastic differential equations were integrated using the EulerMaruyama algorithm [29] specially designed for solving stochastic differential equations. The coherence resonance effect was quantified using normalized variance calculations as in the corresponding experiments. Figure 7 shows the two NV curves obtained for different set points where the autonomous dynamics exhibit fixed point behavior. The three cor- responding time series for one of these NV curves exhibit noise provoked dynamics for little (a), optimum (b), and large (c) levels of superimposed noise. Similar to experimental observations, the maximum regularity of noise induced dynamics is observed for the optimum amplitude of superimposed stochastic fluctuations.

\section{SUMMARY}

The effect of noise has been studied experimentally on both sides of a homoclinic bifurcation. Both destructive and constructive (CR) effects were obtained. The particular role of homoclinity is manifested in the results of Sec. IV involving the emergence of CR. Due to the homoclinic nature of the underlying bifurcation, the frequency of the periodic (period-1) oscillation decreases (going to zero at the bifurcation point) as one approaches the bifurcation point from the oscillatory side, However, the amplitude of the period-1 oscillation remains practically unaltered for the different parameter values (on the oscillatory side) in the vicinity of the homoclinic bifurcation. These two characteristics of the homoclinic bifurcation imply that, while the amplitude of the noise provoked oscillations (CR) is constant, their frequencies vary as a function of the superimposed noise strength.

To reiterate, the present work is in contrast to the results of [27], which report CR for nonexcitable dynamics. Although theoretically noise induced resonance (CR) is expected for excitable dynamics, our results involve the experimental verification of $\mathrm{CR}$ for a chemical system exhibiting excitable behavior. These experiments, apart from their relevance to biological systems, include two factors not usually considered in theoretical papers. First, the electrochemical system has intrinsic chemical noise over which one has no control. Therefore, the emergence of CR experimentally is due to the interplay of three quantities, namely, (a) superimposed noise, (b) intrinsic noise and (c) the deterministic ex- 
citable dynamics. In comparison, the interaction between (a) and (c) results in the emergence of resonances reported for theoretical models. Second, experimental systems exhibit nonstationarity (drift) of the observed dynamical behavior. This results in unavoidable fluctuations of the set point $\left(V_{0}\right)$. The persistence of CR despite these two factors validates the robustness and the generic nature of the noise invoked resonances in real life systems.

\section{ACKNOWLEDGMENT}

The authors acknowledge continuing support from CONACyT (Mexico).
[1] R. Benzi, A. Sutera, and A. Vulpiani, J. Phys. A 14, L453 (1981).

[2] R. Benzi, G. Parisi, A. Sutera, and A. Vulpiani, Tellus 34, 10 (1982).

[3] C. Nicolis and G. Nicolis, Tellus 33, 225 (1981); C. Nicolis, ibid. 34, 1 (1982).

[4] L. Gammaitoni, P. Hanggi, P. Jung, and F. Marchesoni, Rev. Mod. Phys. 70, 223 (1998), and the references within.

[5] P. Jung and G. Mayer-Kress, Phys. Rev. Lett. 74, 2130 (1995).

[6] P. Jung and G. Mayer-Kress, Chaos 5, 458 (1995).

[7] P. Jung and J. W. Shuai, Europhys. Lett. 56, 29 (2001); G. Schmid, I. Goychuk, and P. Hanggi, ibid. 56, 22 (2001).

[8] A. Föster, M. Merget, and F. W. Schneider, J. Phys. Chem. 100, 4442 (1996).

[9] Takashi Amemiya, Takao Ohmori, Mazaru Nakaiawa, and Tomohiko Yamaguchi, J. Phys. Chem. 102, 4537 (1998).

[10] Gerardo J. Escalera Santos and P. Parmananda, Phys. Rev. E 65, 067203 (2002).

[11] P. Hanggi, ChemPhysChem 3, 285 (2002).

[12] A. Longtin, A. Bulsara, and F. Moss, Phys. Rev. Lett. 67, 656 (1991).

[13] B. Lindner, A. Longtin, and A. Bulsara, Neural Comput. 15, 1761 (2003).

[14] B. Lindner, L. Schimansky-Geier, and A. Longtin, Phys. Rev. E 66, 031916 (2002).

[15] A. S. Pikovsky and Jürgen Kurths, Phys. Rev. Lett. 78, 775
(1997).

[16] S. Kandar, J. Wang, and K. Showalter, Nature (London) 391, 770 (1998).

[17] J. D. Murray, Mathematical Biology (Springer, New York, 1993).

[18] S. Grill, V. Zykov, and S. C. Müller, J. Phys. Chem. 100, 19082 (1996).

[19] Antonis Karantonis, Yasuyuki Miyakita, and Seichiro Nakabayashi, Phys. Rev. E 65, 046213 (2002).

[20] Nonlinear Transformations of Stochastic Processes, edited by P. I. Kuznetsov, R. L. Stratonvich, and V. I. Tikkhonov (Pergamon, Oxford, 1965).

[21] P. Hanggi and P. Riseborough, Am. J. Phys. 51, 347 (1983).

[22] W. Ebeling et al., Z. Angew. Math. Mech. 66, 141 (1986).

[23] Yuguo Yu, Wei Wang, Jiafu Wang, and Feng Liu, Phys. Rev. E 63, 021907 (2001).

[24] H. A. Braun et al., Nature (London) 367, 270 (1994).

[25] T. J. Sejnowski, Nature (London) 367, 21 (1995).

[26] Hu Gang, T. Ditzinger, C. Z. Ning, and H. Haken, Phys. Rev. Lett. 71, 807 (1993).

[27] István Z. Kiss, John L. Hudson, Gerardo J. Escalera Santos, and P. Parmananda, Phys. Rev. E 67, 035201 (2003).

[28] Antonis Karantonis and Seichiro Nakabayash, Chem. Phys. Lett. 347, 133 (2001).

[29] Desmond J. Higham, SIAM Rev. 43, 525 (2001). 